\title{
Is diabetic retinopathy affected by diabetes type? A retrospective study using electronic medical record data from patients with latent autoimmune diabetes in adults, type 1 diabetes, and type 2 diabetes
}

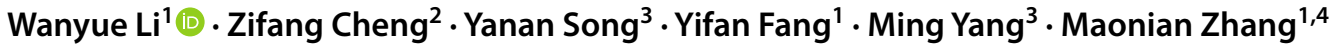

Received: 27 February 2021 / Accepted: 21 May 2021 / Published online: 13 June 2021

(c) The Author(s) 2021

\begin{abstract}
Aims To determine whether the occurrence of diabetic retinopathy (DR) and its related factors are affected by diabetes type (latent autoimmune diabetes in adults [LADA], type 1 diabetes mellitus [T1DM], type 2 diabetes mellitus [T2DM]).

Methods LADA patients were matched for age ( \pm 2 years) and sex to T1DM (1:1) and T2DM (1:2) patients. Retrieved variables included demographic characteristics, diabetes history, laboratory test findings, and history of DR screening, etc. Multiple logistic regression analysis was applied to identify influencing factors of DR. A decision tree was used to explore interactions between diabetes type and other influencing factors of DR.

Results We included 110 LADA, 101 T1DM, and 220 T2DM patients. DR prevalence was $26.4 \%$ in LADA patients, lower than that in T1DM $(50.5 \%)$ and T2DM $(47.7 \%)$ patients $(P<0.001)$. Logistic regression analysis demonstrated that diabetes duration $(\mathrm{OR}=1.15,95 \% \mathrm{CI}: 1.1-1.26, P<0.001)$ and diabetic nephropathy $(\mathrm{DN})(\mathrm{OR}=42.39,95 \% \mathrm{CI}: 10.88-165.11$, $P<0.001)$ were independent risk factors for $\mathrm{DR}$, and regular DR screening $(\mathrm{OR}=0.33,95 \% \mathrm{CI}$ : $0.16-0.69, P=0.003)$ was an independent protective factor. Decision tree analysis showed that in patients without DN with a diabetes duration of at least 10.5 years, T1DM and LADA patients had a higher incidence of DR than T2DM patients $(72.7 \%$ vs. $55.1 \%)$.

Conclusions The prevalence of DR in diabetes patients was affected by diabetes duration, DN occurrence, and regular DR screening. Diabetes type indirectly affects DR occurrence through its interaction with diabetes duration and DN. Correct LADA diagnosis is necessary, and DR screening needs to be well-implemented.
\end{abstract}

Keywords Diabetic retinopathy · Diabetic nephropathy $\cdot$ Latent autoimmune diabetes in adults · Type 2 diabetes mellitus · Type 1 diabetes mellitus $\cdot$ Influencing factors

This article belongs to the topical collection Eye Complications of Diabetes, managed by Giuseppe Querques.

Wanyue Li and Zifang Cheng contributed equally to this work.

Maonian Zhang

zmn301@sina.com

1 Medical School of Chinese PLA, Beijing, China

2 The Northern Medical District of Chinese PLA General Hospital, Beijing, China

3 Medical Big Data Research Center, Medical Innovation Research Division of Chinese People's Liberation Army General Hospital, Beijing, China

4 Department of Ophthalmology, Chinese PLA General Hospital, Beijing, China

\section{Introduction}

Latent autoimmune diabetes in adults (LADA) is a subtype of type 1 diabetes mellitus (T1DM) [1,2]. Patients with LADA do not initially require insulin and have the same clinical characteristics as type 2 diabetes mellitus (T2DM) patients at diagnosis. Within a few years, autoimmune imbalance leads to progressive pancreatic $\beta$-cell dysfunction and insulin dependency [3]. An estimated $4-14 \%$ of LADA patients are initially diagnosed with T2DM [4]. Compared with T1DM, LADA is not uncommon.

Diabetic retinopathy (DR) is a leading cause of vision impairment and blindness in the working-age population globally [5,6]. Although enormous studies have focused on the epidemiology and risk factors of DR, data on DR in patients with LADA are limited. Only a few studies have introduced the clinical characteristics of DR in LADA 
patients, and most of them have only focused on its prevalence. For instance, in a comparison of the prevalence and incidence of chronic complications between LADA and T2DM patients, Myhill et al. [7] found that there was a similar prevalence and incidence of retinopathy in the two groups ( $P=0.22$ and 0.64 , respectively). In another study aimed at analyzing the relationship between glycaemic variability and DR, researchers reported that no metrics related to glycaemic variability were identified as independent risk factors of DR (standard deviation: $P=0.175$; coefficient of variation: $P=0.769$; mean amplitude of glycaemic excursions: $P=0.388$ ) in LADA patients [8]; however, other risk factors were not examined. In a study by Park et al. [9] that recruited 432 newly diagnosed diabetes mellitus patients, six patients diagnosed with LADA demonstrated proliferative diabetic retinopathy (PDR) as an initial sign. This suggests that retinopathy may develop rapidly in LADA patients.

Therefore, to better manage LADA patients, this study was designed to determine whether the occurrence of DR and its related factors are affected by diabetes type (T1DM, LADA, and T2DM).

\section{Materials and method}

\section{Data collection}

In this study, the clinical data of patients diagnosed with LADA were extracted from the Chinese PLA General Hospital electronic medical record system from 1 January 2013 to 31 October 2020. Age- ( \pm 2 years) and sex-matched patients with T1DM and T2DM were randomly selected in a 1:1 and 2:1 ratio, respectively, in the same period.

\section{Inclusion and exclusion criteria}

Included patients were either hospitalized in ophthalmology or had received ophthalmic consultation. Patients with missing data or with cataract, keratitis, corneal speckles, and other eye diseases that affect fundus examination were excluded from the study.

\section{Data extraction criteria}

The first record of measurement upon admission for each variable was extracted. Diagnostic information of the diseases was extracted from the discharge diagnosis records. Retrieved variables included demographic characteristics (sex and age), history of diabetes (diabetes typing, duration, and number of episodes of ketoacidosis), diabetes complications (diabetic nephropathy, neuropathy, retinopathy, and coronary heart disease), hypertension, laboratory parameters (fasting blood glucose, postprandial blood glucose, glycosylated hemoglobin [HbA1c], triglycerides, total cholesterol, high-density lipoproteins, low-density lipoproteins, urea, serum creatinine, and urinary microalbumin/ creatinine), physical indicators (weight, height, and systolic and diastolic blood pressure), DR screening history (absence of DR screening, regular DR screening, and number of years until the first fundus examination after diabetes diagnosis), and smoking history. Body mass index (BMI) was calculated as the weight $(\mathrm{kg})$ divided by height in meters squared $\left(\mathrm{m}^{2}\right)$.

\section{Diagnostic criteria}

The diagnostic criteria for LADA in our hospital are as follows: $>30$ years of age, positivity for glutamic acid decarboxylase autoantibodies (GADA), and no requirement of insulin within at least 6 months after diagnosis. The diagnostic criteria for T1DM and T2DM followed those set forth by the 2003 American Diabetes Association (ADA) guidelines [10]. DR was diagnosed according to the International Clinical Diabetic Retinopathy Severity Scale [11] by using color fundus photography and indirect ophthalmoscopy when the pupils were dilated. Fundus examination, image reading, and diagnosis were performed by at least two experienced ophthalmologists for each patient, whether directly admitted to the ophthalmology department or for ophthalmic consultation in other departments.

This retrospective study was approved by the Chinese PLA General Hospital clinical research ethics committee (No. S2019-326-02, February 25, 2020) and adhered to the tenets of the Declaration of Helsinki.

\section{Statistical analyses}

In this study, IBM SPSS, version 23.0 (IBM Corp., Armonk, NY, USA) was used for statistical analyses. Categorical variables were expressed as numbers and percentages. Continuous variables were expressed as means with standard deviations. The Student's $t$ test and analysis of variance (ANOVA) were performed for continuous variables to assess the statistical significance of differences between groups. The chi-square test was used to compare categorical variables. Multiple logistic regression analysis was applied to the whole dataset to identify the influencing factors of DR. The variables included in the logistic regression analysis were determined based on the $P$ value in the univariate analysis and applying collinearity diagnostics. The Hosmer-Lemeshow test was used to assess the goodness of fit of the model. A decision tree was used to explore the interactions between diabetes types and other influencing factors with respect to DR. A value of $P<0.05$ was considered statistically significant. 


\section{Results}

\section{Baseline analysis of three types of diabetes}

A total of 110 LADA, 101 T1DM, and 220 T2DM patients were enrolled in the analysis. The clinical characteristics and laboratory test results in each group are shown in Table 1. Patients with LADA, T1DM, and T2DM were similar in terms of age, sex, smoking history, and levels of total cholesterol, urea, serum creatinine, fasting blood glucose, and postprandial blood glucose. Diabetes duration was shorter in LADA patients than in those with T1DM and $\mathrm{T} 2 \mathrm{DM}(6.01 \pm 5.65$ vs. $10.70 \pm 9.55$ vs. $8.97 \pm 7.15$, $P<0.001)$ when each group was matched by age.

Patients with T2DM had higher BMI scores; systolic blood pressure; diastolic blood pressure; and levels of triglycerides, low-density lipoprotein, and lower high-density lipoprotein than LADA and T1DM patients. There were no significant differences in those variables between the LADA and T1DM groups. Patients with T2DM were less likely to develop ketoacidosis repeatedly over the course of the disease (T1DM 17.8\%, T2DM 2.3\%, LADA 9.1\%; $P<0.001$ ). The prevalence of DR was $26.4 \%$ in LADA patients, which was lower than that in T1DM and T2DM patients $(50.5 \%$ and $47.7 \%$, respectively; $P<0.001$ ).

Table 1 Clinical characteristics and laboratory tests of patients with T1DM, T2DM and LADA

\begin{tabular}{|c|c|c|c|c|}
\hline Variables & Type 1 diabetes $(n=101)$ & Type 2 diabetes $(n=220)$ & $\operatorname{LADA}(n=110)$ & $P$ \\
\hline Male, $n(\%)$ & $60(59.4 \%)$ & $130(59.1 \%)$ & $65(59.1 \%)$ & 0.998 \\
\hline Age, years & $45.64 \pm 11.27$ & $46.50 \pm 10.93$ & $46.11 \pm 11.03$ & 0.81 \\
\hline Duration of diabetes, years & $10.70 \pm 9.55 a$ & $8.97 \pm 7.15 \mathrm{a}$ & $6.01 \pm 5.65 b$ & $<0.001 * *$ \\
\hline BMI, $\mathrm{kg} / \mathrm{m}^{2}$ & $22.18 \pm 2.88 \mathrm{a}$ & $26.32 \pm 3.90 \mathrm{~b}$ & $21.60 \pm 3.03 \mathrm{a}$ & $<0.001 * *$ \\
\hline Systolic blood pressure, $\mathrm{mmHg}$ & $127.16 \pm 19.73 \mathrm{a}$ & $136.60 \pm 22.40 \mathrm{~b}$ & $121.40 \pm 19.51 \mathrm{a}$ & $<0.001 * *$ \\
\hline Diastolic blood pressure, $\mathrm{mmHg}$ & $74.03 \pm 11.51 \mathrm{a}$ & $81.06 \pm 14.34 b$ & $73.65 \pm 11.28 \mathrm{a}$ & $<0.001 * *$ \\
\hline Total cholesterol, mmol/L & $4.19 \pm 0.98$ & $4.49 \pm 1.34$ & $4.25 \pm 1.09$ & 0.068 \\
\hline Triglyceride, $\mathrm{mmol} / \mathrm{L}$ & $1.17 \pm 0.72 \mathrm{a}$ & $2.34 \pm 2.44 b$ & $1.04 \pm 0.92 \mathrm{a}$ & $<0.001 * *$ \\
\hline High-density lipoproteins, $\mathrm{mmol} / \mathrm{L}$ & $1.44 \pm 0.47 \mathrm{a}$ & $1.07 \pm 0.29 b$ & $1.47 \pm 0.45 \mathrm{a}$ & $<0.001 * *$ \\
\hline Low-density lipoproteins, mmol/L & $2.50 \pm 0.85 \mathrm{a}$ & $2.83 \pm 0.97 b$ & $2.56 \pm 0.99 a$ & $0.005 * *$ \\
\hline Urea, $\mathrm{mmol} / \mathrm{L}$ & $7.41 \pm 6.84$ & $7.26 \pm 5.86$ & $5.90 \pm 3.66$ & 0.08 \\
\hline Serum creatinine, $\mu \mathrm{mol} / \mathrm{L}$ & $137.67 \pm 202.21$ & $128.95 \pm 195.60$ & $85.06 \pm 127.21$ & 0.065 \\
\hline Urinary microalbumin/creatinine & $181.75 \pm 373.09 \mathrm{a}$ & $162.00 \pm 299.94 \mathrm{a}$ & $36.93 \pm 103.24 b$ & $<0.001 * *$ \\
\hline Fasting blood glucose, mmol/L & $9.66 \pm 3.92$ & $8.89 \pm 3.14$ & $9.43 \pm 3.79$ & 0.144 \\
\hline Postprandial blood glucose, $\mathrm{mmol} / \mathrm{L}$ & $14.40 \pm 5.33$ & $13.07 \pm 4.40$ & $13.74 \pm 5.40$ & 0.073 \\
\hline $\mathrm{HbA} 1 \mathrm{c}, \%$ & $8.79 \pm 1.95 \mathrm{a}$ & $8.50 \pm 2.26 a$ & $9.52 \pm 2.31 b$ & $<0.001 * *$ \\
\hline Ketoacidosis, $n(\%)$ & & & & $<0.001 * *$ \\
\hline Never & $35(34.7 \%) \mathrm{a}$ & $188(85.5 \%) b$ & $52(47.3 \%) \mathrm{a}$ & \\
\hline 1 time & $48(47.5 \%) \mathrm{a}$ & $27(12.3 \%) \mathrm{b}$ & $48(43.6 \%) \mathrm{a}$ & \\
\hline More than 1 time & $18(17.8 \%) \mathrm{a}$ & $5(2.3 \%) \mathrm{b}$ & $10(9.1 \%) \mathrm{a}$ & \\
\hline Smoking, $n(\%)$ & $37(36.6 \%)$ & $91(41.4 \%)$ & $39(35.5 \%)$ & 0.515 \\
\hline Regular DR screening, $n(\%)$ & $30(29.7 \%) \mathrm{a}$ & $32(14.5 \%) b$ & $42(38.2 \%) \mathrm{a}$ & $<0.001 * *$ \\
\hline Never DR screening, $n(\%)$ & $46(45.5 \%)$ & $116(52.7 \%)$ & $57(51.8 \%)$ & 0.475 \\
\hline First DR screening, years & $7.97 \pm 7.77 \mathrm{a}$ & $6.91 \pm 6.77 \mathrm{a}$ & $4.53 \pm 4.94 b$ & $<0.001 * *$ \\
\hline Hypertension, $n(\%)$ & $31(30.7 \%) \mathrm{a}$ & $122(55.5 \%) \mathrm{b}$ & $20(18.2 \%) \mathrm{a}$ & $<0.001 * *$ \\
\hline Coronary heart disease, $n(\%)$ & $17(16.8 \%) \mathrm{a}$ & $14(6.4 \%) b$ & $14(12.7 \%) \mathrm{a}, \mathrm{b}$ & $0.011^{*}$ \\
\hline Diabetic nephropathy, $n(\%)$ & $21(20.8 \%) a, b$ & $54(24.5 \%) \mathrm{b}$ & $12(10.9 \%) \mathrm{a}$ & $0.014^{*}$ \\
\hline Diabetic neuropathy, $n(\%)$ & $31(30.7 \%) \mathrm{a}, \mathrm{b}$ & $93(42.3 \%) b$ & $26(23.6 \%) \mathrm{a}$ & $0.002 * *$ \\
\hline Diabetic retinopathy, $n(\%)$ & $51(50.5 \%) \mathrm{a}$ & $105(47.7 \%) \mathrm{a}$ & $29(26.4 \%) b$ & $<0.001 * *$ \\
\hline
\end{tabular}

Values are expressed as the mean $\pm \mathrm{SD}$ or number (percentages).

T1DM, type 1 diabetes mellitus; LADA, latent autoimmune diabetes in adults; T2DM, type 2 diabetes mellitus; BMI body mass index; HbA1c, glycosylated hemoglobin; DR diabetic retinopathy.

$* P<0.05$ for multi-group variance analysis or chi-square test; $* * P<0.01$ for multi-group variance analysis or chi-square test; a/b, for each variable, there was no significant difference between the groups marked with the same letter 
Only $14.5 \%$ of T2DM patients underwent regular DR screening every year; this proportion was significantly lower than those in T1DM and LADA patients $(29.7 \%$ and $38.2 \%$, respectively; $P<0.001$ ). The first DR screening was performed earlier in LADA patients than in T1DM and T2DM patients. Almost half of the diabetes patients never underwent a fundus examination after diagnosis (T1DM $45.5 \%$, T2DM 52.7\%, LADA 51.8\%), which means that they received their first fundus examination during this hospitalization.

\section{Influencing factors of diabetic retinopathy}

All diabetes patients were divided into two subgroups: the DR group and the non-DR (NDR) group. According to the results of univariate analysis, patients with DR had a longer diabetes duration $(13.09 \pm 7.75$ years vs. $5.25 \pm 5.53$ years, $P<0.001$ ), higher fasting blood glucose levels $(9.41 \pm 3.80 \mathrm{mmol} / \mathrm{L}$ vs. $9.061 \pm 3.27 \mathrm{mmol} / \mathrm{L}$, $P=0.047)$, higher BMI $\left(24.35 \pm 3.63 \mathrm{~kg} / \mathrm{m}^{2}\right.$ vs. $23.99 \pm 4.45 \mathrm{~kg} / \mathrm{m}^{2}, P=0.033$ ), higher low-density lipoprotein levels $(2.80 \pm 1.07 \mathrm{mmol} / \mathrm{L}$ vs. $2.60 \pm 0.87 \mathrm{mmol} / \mathrm{L}$,
$P=0.007)$, higher urinary microalbumin/creatinine levels $(266.95 \pm 373.69 \mathrm{mg} / \mathrm{g}$ vs. $35.26 \pm 140.81 \mathrm{mg} / \mathrm{g}, P<0.001)$, and a lower proportion of regular DR screening (10.3\% vs. $34.6 \%, P<0.001)$ than those without DR. Diabetic nephropathy and neuropathy were also more common in patients with DR than in those without ( $45.4 \%$ vs. $1.2 \%, P<0.001$ and $45.4 \%$ vs. $26.8 \%, P<0.001$, respectively). The univariate analysis results are shown in Table 2 .

Logistic regression analysis was performed on all diabetes patients to determine the influencing factors of DR. According to the results of univariate analysis (variables with $P$ values $<0.05$ ) and clinical experience, 10 variables were selected, including diabetes duration, BMI, fasting blood glucose, low-density lipoprotein, urinary microalbu$\mathrm{min} /$ creatinine, hypertension, regular DR screening, diabetic nephropathy, neuropathy, and type of diabetes. Considering that it can reflect renal function sensitively, urinary microalbumin/creatinine was chosen as the representative of renal function in laboratory tests. Before logistic regression analysis, collinearity diagnostics were performed. As shown in Online Resource 1 , there was no collinearity among the 10 variables to be included in the logistic regression analysis.
Table 2 Results of univariate analysis

\begin{tabular}{|c|c|c|c|}
\hline Variables & $\operatorname{NDR}(n=246)$ & $\mathrm{DR}(n=185)$ & $P$ \\
\hline Male, $n(\%)$ & $155(63.0 \%)$ & $100(54.1 \%)$ & 0.061 \\
\hline Age, years & $44.15 \pm 11.23$ & $48.92 \pm 10.13$ & 0.09 \\
\hline Duration of diabetes, years & $5.25 \pm 5.53$ & $13.09 \pm 7.75$ & $<0.001 * *$ \\
\hline BMI, $\mathrm{kg} / \mathrm{m}^{2}$ & $23.99 \pm 4.45$ & $24.35 \pm 3.63$ & $0.033^{*}$ \\
\hline Systolic blood pressure, $\mathrm{mmHg}$ & $124.77 \pm 20.64$ & $138.14 \pm 21.56$ & 0.288 \\
\hline Diastolic blood pressure, $\mathrm{mmHg}$ & $76.25 \pm 13.45$ & $79.22 \pm 13.30$ & 0.354 \\
\hline Total cholesterol, $\mathrm{mmol} / \mathrm{L}$ & $4.27 \pm 1.06$ & $4.47 \pm 1.37$ & 0.068 \\
\hline Triglyceride, $\mathrm{mmol} / \mathrm{L}$ & $1.69 \pm 2.02$ & $1.80 \pm 1.83$ & 0.56 \\
\hline High-density lipoproteins, mmol/L & $1.28 \pm 0.46$ & $1.24 \pm 0.38$ & 0.133 \\
\hline Low-density lipoproteins, mmol/L & $2.60 \pm 0.87$ & $2.80 \pm 1.07$ & $0.007 * *$ \\
\hline Urea, mmol/L & $5.28 \pm 2.31$ & $9.17 \pm 7.70$ & $<0.001 * *$ \\
\hline Serum creatinine, $\mu \mathrm{mol} / \mathrm{L}$ & $72.27 \pm 55.41$ & $182.99 \pm 259.27$ & $<0.001^{* *}$ \\
\hline Urinary microalbumin/creatinine, $\mathrm{mg} / \mathrm{g}$ & $35.26 \pm 140.81$ & $266.95 \pm 373.69$ & $<0.001^{*}$ \\
\hline Fasting blood glucose, $\mathrm{mmol} / \mathrm{L}$ & $9.061 \pm 3.27$ & $9.41 \pm 3.80$ & $0.047^{*}$ \\
\hline Postprandial blood glucose, $\mathrm{mmol} / \mathrm{L}$ & $13.70 \pm 5.04$ & $13.37 \pm 4.75$ & 0.432 \\
\hline $\mathrm{HbA} 1 \mathrm{c}, \%$ & $9.05 \pm 2.22$ & $8.53 \pm 2.23$ & 0.517 \\
\hline Smoking, $n(\%)$ & $103(41.9 \%)$ & $64(34.6 \%)$ & 0.125 \\
\hline Regular DR screening, $n(\%)$ & $85(34.6 \%)$ & $19(10.3 \%)$ & $<0.001 * *$ \\
\hline Never DR screening, $n(\%)$ & $135(54.9 \%)$ & $84(45.4 \%)$ & 0.052 \\
\hline First DR screening, years & $3.67 \pm 4.31$ & $10.39 \pm 7.41$ & $<0.001^{* *}$ \\
\hline Hypertension, $n(\%)$ & $71(28.9 \%)$ & $102(55.1 \%)$ & $<0.001 * *$ \\
\hline Coronary heart disease, $n(\%)$ & $23(9.3 \%)$ & $22(11.9 \%)$ & 0.393 \\
\hline Diabetic nephropathy, $n(\%)$ & $3(1.2 \%)$ & $84(45.4 \%)$ & $<0.001^{* *}$ \\
\hline Diabetic neuropathy, $n(\%)$ & $66(26.8 \%)$ & $84(45.4 \%)$ & $<0.001 * *$ \\
\hline
\end{tabular}

Values are expressed as the mean $\pm \mathrm{SD}$ or number (percentages).

BMI body mass index; HbA1c, glycosylated hemoglobin; DR diabetic retinopathy.

${ }^{*} P<0.05$ for $T$ test or chi-square test; ${ }^{*} P<0.01$ for $T$ test or chi-square test 
The associations between DR and the above 10 influencing factors in diabetes patients were assessed using multivariable binary logistic regression, and the calculated odd ratios (ORs) are presented in Table 3. Diabetes duration $(\mathrm{OR}=1.15,95 \%$ confidence interval $[\mathrm{CI}]: 1.1-1.26$, $P<0.001)$ and diabetic nephropathy $(\mathrm{OR}=42.39,95 \% \mathrm{CI}$ : $10.88-165.11, P<0.001)$ were observed to be independent risk factors for DR, and regular DR screening $(\mathrm{OR}=0.33$, 95\% CI: $0.16-0.69, P=0.003$ ) was an independent protective factor. The Hosmer-Lemeshow test showed that the model seemed to fit well $(P=0.178)$.

\section{Influence of diabetes type on the occurrence of diabetic retinopathy}

To explore whether there were interactions between diabetes type and the risk and protective factors that influenced the occurrence of DR, decision tree analysis was performed. Type of diabetes and three influencing factors (diabetes duration, diabetic nephropathy, and regular DR screening) were included in the decision tree model. In diabetes patients without diabetic nephropathy with a diabetes duration of at least 10.5 years, results showed that those diagnosed with T1DM and LADA had a higher incidence of DR than those with T2DM $(72.7 \%$ vs. $55.1 \%)$. The decision tree is detailed in Fig. 1. The confusion matrix of the decision tree model is shown in Online Resource 2. According to the confusion matrix, the accuracy, specificity, sensitivity, and the F1 value of the model were calculated to be $81.7 \%, 85 \%, 77.3 \%$, and 0.78 , respectively. The receiver operator characteristic (ROC) curve of the decision tree model is shown in Fig. 2.

Table 3 Results of multivariable logistic regression analysis

\begin{tabular}{lll}
\hline Variables & OR $(95 \% \mathrm{CI})$ & $P$ \\
\hline Type of diabetes & & 0.323 \\
T2DM & $0.64(0.30,1.36)$ & 0.246 \\
LADA & $0.57(0.26,1.26)$ & 0.166 \\
Duration of diabetes & $1.15(1.10,1.21)$ & $0.000^{* *}$ \\
Hypertension (1) & $0.65(0.34,1.22)$ & 0.176 \\
BMI & $1.03(0.95,1.11)$ & 0.507 \\
Fasting blood glucose & $1.06(0.98,1.16)$ & 0.137 \\
Low-density lipoproteins & $1.30(0.97,1.75)$ & 0.084 \\
Diabetic nephropathy (1) & $42.39(10.88,165.11)$ & $0.000^{* *}$ \\
Peripheral neuropathy (1) & $1.60(0.92,2.80)$ & 0.099 \\
Regular DR screening (1) & $0.33(0.16,0.69)$ & $0.003 * *$ \\
Urinary microalbumin/creatinine & $1.00(1.00,1.00)$ & 0.136 \\
\hline
\end{tabular}

Hosmer-Lemeshow test: $P=0.178$

T1DM, type 1 diabetes mellitus; LADA, latent autoimmune diabetes in adults; T2DM, type 2 diabetes mellitus; BMI body mass index; DR diabetic retinopathy; OR, odd ratio; CI, confidence interval.

$* P<0.05 ; * * P<0.01$
The model had an area under the curve (AUC) of 0.811 (95\% CI: 0.768-0.855), which was considered to indicate good performance.

\section{Discussion}

In this study, age- ( \pm 2 years $)$ and sex-matched patients with T1DM, T2DM, and LADA were enrolled to investigate the relationship between diabetes type and occurrence of DR. DR threatens visual function in $14.77 \%-22.43 \%$ of patients with diabetes in China [12]. The high prevalence of DR in our study population (T1DM 50.5\%, T2DM 47.7\%, LADA $26.4 \%$ ) might be explained by the fact that our hospital is one of the top general hospitals in the country, and the conditions of the in patients are complicated. In a study conducted in Korea, the results showed similar prevalence rates of DR in the same three patient populations [13]. However, Lu et al. [14] reported that the DR prevalence was higher in patients with T2DM than in patients with LADA ( $25 \%$ vs. $20.3 \%$, $P=0.033$ ). In our study, patients in the LADA group had a shorter diabetes duration and a lower DR prevalence than T1DM and T2DM patients. As seen in the logistic regression analysis, diabetes duration was an independent risk factor for DR. This finding is supported by findings from previous studies $[15,16]$. Therefore, the shortest mean diabetes duration in the LADA group was inferred to be related to the lowest DR prevalence seen in this group, as compared to those in the remaining two groups.

Remarkably, LADA patients had higher DR screening rates and underwent the first fundus examination at an earlier time-point than those in the other two groups. The reason for these differences is unclear. Furthermore, in the logistic regression analysis, regular DR screening was an independent protective factor of $\mathrm{DR}(\mathrm{OR}=0.33,95 \% \mathrm{CI}$ : $0.16-0.69, P=0.003)$. We have not been able to find previous studies that had similar content. The ADA recommends dilated and comprehensive eye examinations every 1-2 years for diabetes patients without evidence of retinopathy, and more frequent examinations are necessary if any level of DR is present or if sight is threatened [17]. However, not every patient undergoes annual dilated and comprehensive eye examinations. In our study, almost half of the diabetes patients never underwent a fundus examination after diagnosis (T1DM 45.5\%, T2DM 52.7\%, LADA 51.8\%). Owing to the preventive effects of therapy and the fact that patients with PDR or macular oedema may be asymptomatic, the ADA emphasized that DR screening should be strongly supported [17]. Additionally, our results suggest that performing screening early and regularly has a positive effect on preventing the occurrence of DR. This may be due to greater awareness of health management in these patients, as well as 


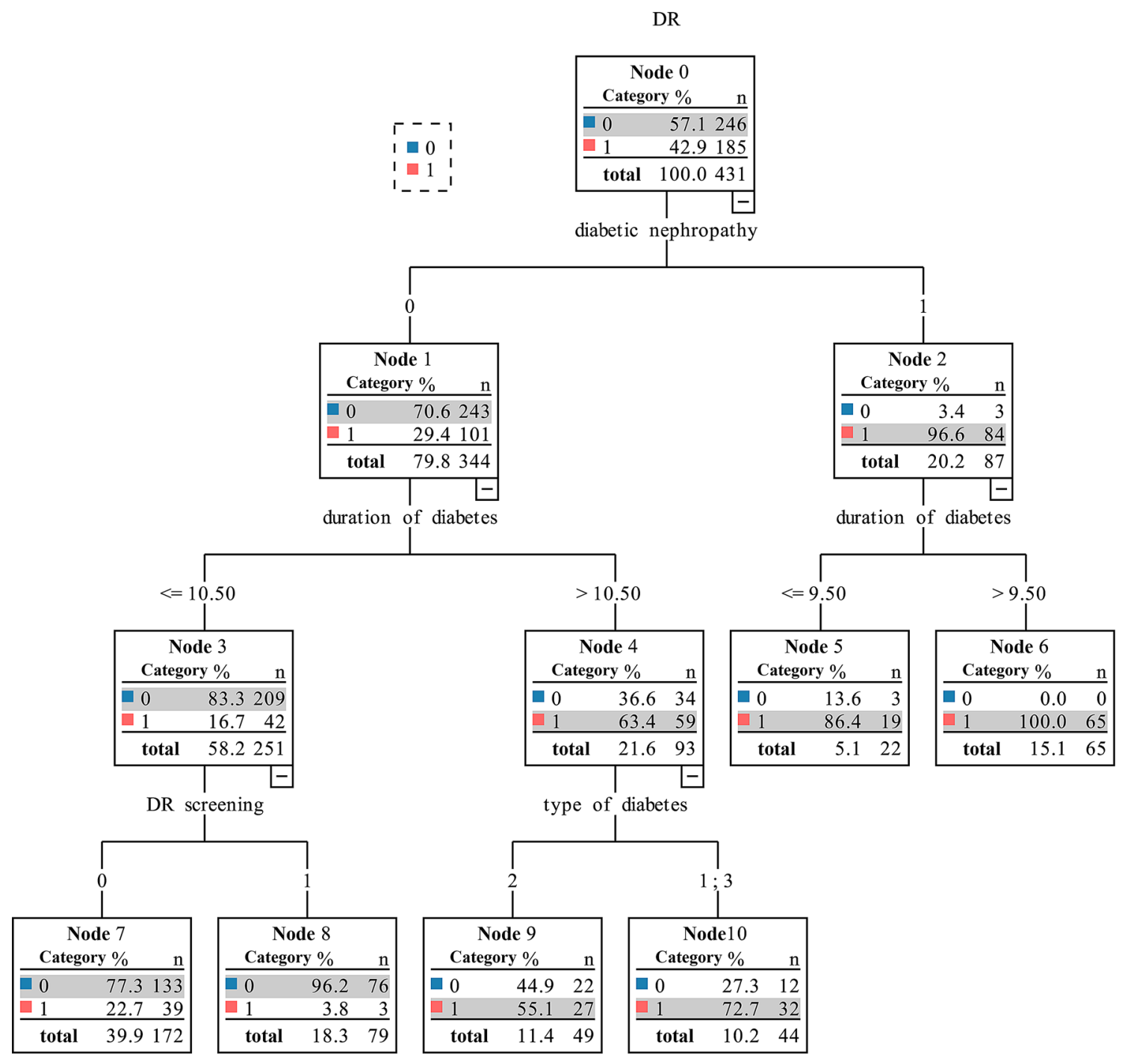

Fig. 1 Decision tree analysis for predicting diabetic retinopathy. In the figure, the numbers at each node represent the true values, and the categories with a gray background represent outcomes predicted by the model. A blue square of 0 and a red square of 1 represent the absence and presence of diabetic retinopathy, respectively. 431 diabetic patients were included in the analysis, of whom 185 had diabetic retinopathy and 246 did not. Of the patients predicted to be negative for diabetic retinopathy by the model (negative predictive

greater access to physician advice during screening. Given the low DR screening rate, doctors have much work to do.

In the case of diabetic nephropathy, the DR group had worse kidney function and a higher incidence of DN than the NDR group. It has been widely confirmed that DN is associated with DR in diabetes patients [18-22]. Similarly, the current study found that diabetic nephropathy was an independent risk factor of DR. Both the retinas and kidneys are organs supplied by the microvasculature, which is sensitive to fluctuations in blood flow [23]. The progression of retinopathy and nephropathy affects each other, supporting value), 243/344 (70.6\%) did not have diabetic retinopathy; of the patient predicted to be positive for diabetic retinopathy by the model (positive predictive value), 84/87 (96.6\%) had diabetic retinopathy. The interpretation of the rest of the nodes is similar. With respect to subdivisions according to diabetes type, 1 represents type 1 diabetes mellitus, 2 represents type 2 diabetes mellitus, and 3 represents latent autoimmune diabetes in adults. DR, diabetic retinopathy (color figure online)

the view of a shared etiological basis and emphasizing the need for a multidisciplinary approach to diabetes care [24].

To investigate whether the occurrence of DR and its related factors are affected by the type of diabetes (T1DM, LADA, and T2DM), diabetes type was included in multivariable logistic regression analysis. We found that diabetes type was not an independent influencing factor of DR. This was consistent with the results of the Fremantle Diabetes Study. In their logistic regression model, diabetes duration, $\mathrm{HbA} 1 \mathrm{c}$, systolic blood pressure, and current smoking were each significantly and independently predictive of retinopathy, but 


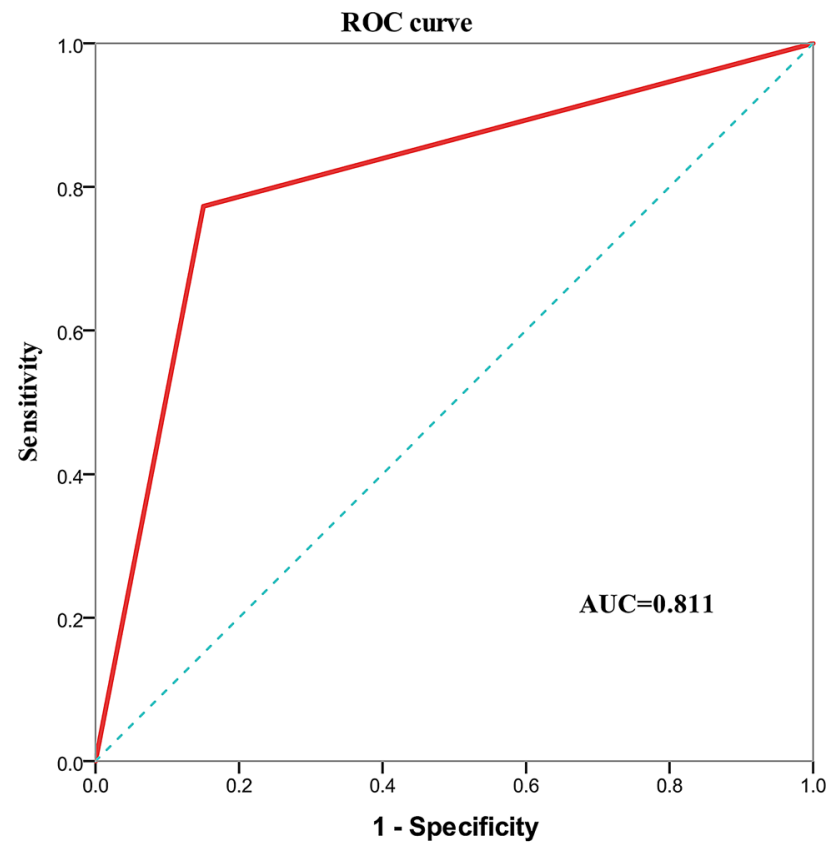

Fig. 2 Receiver-operating characteristic curve of the decision tree model for predicting diabetic retinopathy. The receiver-operating characteristic curve was generated by calculating the sensitivity and specificity. The Y-axis shows the sensitivity (true positive rate) for predicting diabetic retinopathy. The $\mathrm{X}$-axis shows the $1-$ specificity (false positive rate) of the diabetic retinopathy prediction model. The red line represents the performance of the decision tree model. The area under the curve for this model is 0.811 . ROC, receiver operator characteristic; AUC, area under the curve (color figure online)

GADA status was not [15]. We further explored whether the type of diabetes affected the occurrence of DR through interaction with other influencing factors. The results of the decision tree analysis indicated that there was an interaction between diabetes type and diabetes duration in patients without nephropathy. In patients with T1DM and LADA with a long disease course, more attention should be paid to health management, and follow-up regarding fundus health should be strengthened.

It has been widely reported that patients with LADA generally have lower triglyceride levels; higher highdensity lipoprotein cholesterol levels; and lower BMI, waist-to-hip ratio, and blood pressure than those with T2DM [25]. The prevalence of metabolic syndrome was reported to be significantly higher in T2DM patients than in patients with LADA or T1DM [26]. The baseline analysis of our study revealed similar results. In another study, the inverse association between simultaneous positivity for antibodies against islet cell cytoplasmic antigens (ICA), glutamic acid decarboxylase enzyme (GAD), and tyrosine phosphatase-like transmembrane glycoprotein (IA2) and metabolic syndrome and its components present in LADA patients might imply that LADA patients are phenotypically closer to T1DM patients [27]. In our study, the history of ketoacidosis was similar between LADA and T1DM patients, but it was worse in these two populations than in T2DM patients. LADA patients also had the highest HbA1c levels among the three groups. LADA patients tend to have worse glycaemic control than patients with T2DM [4]. Therefore, it is necessary to reduce the misdiagnosis rate of LADA patients, strictly follow up on their islet function, and formulate an appropriate glycaemic control regimen. Correct diagnosis is the cornerstone of health management and reduction of complications.

A strength of this study was the analysis of influencing factors of DR in patients with LADA, T1DM, and T2DM based on an age- and sex-matched dataset. The interactions between diabetes type and the influencing factors for DR were further explored, which have rarely been reported in previous studies and provide a reference for future research. Admittedly, this study has some limitations. The sample size was not large enough for a more detailed analysis on influencing factors. In addition, this was a retrospective study, and the causal relationship between the variables and DR needs to be explored in future prospective studies and randomized clinical trials with sufficiently long follow-up periods.

In conclusion, this study revealed that the clinical features of LADA are closer to those of T1DM, and patients with LADA present with worse glycaemic control than patients with T2DM. Diabetes type was not an independent influencing factor of DR, but among diabetes patients without diabetic nephropathy with a diabetes duration of at least 10.5 years, T1DM and LADA patients had a higher incidence of DR than those with T2DM. Regular DR screening is an independent protective factor of DR. Correct diagnosis of LADA is necessary, and DR screening needs to be well-implemented.

Supplementary Information The online version contains supplementary material available at https://doi.org/10.1007/s00592-021-01748-0.

Acknowledgements The authors thank the computer office of Chinese PLA general hospital for its assistance in extracting clinical data.

Authors' contributions WL designed the data extraction standard and analysis scheme and drafted the manuscript. ZC checked and interpreted the data and drafted the manuscript. YS analyzed the data and reviewed the manuscript for important intellectual content. MY acquired, and cleaned the data and reviewed the manuscript for important intellectual content. YF acquired and checked the data. MZ conceived and designed the study and reviewed the manuscript for important intellectual content. All the authors approved the final version of the manuscript submitted.

Funding No funding was received for conducting this study.

Data availability The dataset is available from the corresponding author on reasonable request. 
Code availability The code is available from the corresponding author on reasonable request.

\section{Declarations}

Conflicts of interest The authors declare no conflicts of interest.

Ethics approval This study was approved by the Chinese PLA General Hospital clinical research ethics committee (No. S2019-326-02, February 25,2020$)$.

Informed consent This was a retrospective study in which de-privatized data was extracted from the electronic medical record system so informed consent were not needed.

Open Access This article is licensed under a Creative Commons Attribution 4.0 International License, which permits use, sharing, adaptation, distribution and reproduction in any medium or format, as long as you give appropriate credit to the original author(s) and the source, provide a link to the Creative Commons licence, and indicate if changes were made. The images or other third party material in this article are included in the article's Creative Commons licence, unless indicated otherwise in a credit line to the material. If material is not included in the article's Creative Commons licence and your intended use is not permitted by statutory regulation or exceeds the permitted use, you will need to obtain permission directly from the copyright holder. To view a copy of this licence, visit http://creativecommons.org/licenses/by/4.0/.

\section{References}

1. Palmer JP, Hampe CS, Chiu H, Goel A, Brooks-Worrell BM (2005) Is latent autoimmune diabetes in adults distinct from type 1 diabetes or just type 1 diabetes at an older age. Diabetes 54(Suppl 2):S62-67. https://doi.org/10.2337/diabetes.54.suppl_2.s62

2. Redondo MJ (2013) LADA: time for a new definition. Diabetes 62(2):339-340. https://doi.org/10.2337/db12-1171

3. ADA (1997) Report of the expert committee on the diagnosis and classification of diabetes mellitus. Diabetes Care 20(7):11831197. https://doi.org/10.2337/diacare.20.7.1183

4. Laugesen E, Østergaard JA, Leslie RD, Workshop DDA, Speakers W (2015) Latent autoimmune diabetes of the adult: current knowledge and uncertainty. Diabetes Med 32(7):843-852. https:// doi.org/10.1111/dme. 12700

5. Frank RN (2004) Diabetic retinopathy. N Engl J Med 350(1):4858. https://doi.org/10.1056/NEJMra021678

6. Cheung N, Mitchell P, Wong TY (2010) Diabetic retinopathy. Lancet 376(9735):124-136. https://doi.org/10.1016/S01406736(09)62124-3

7. Myhill P, Davis WA, Bruce DG, Mackay IR, Zimmet P, Davis TM (2008) Chronic complications and mortality in community-based patients with latent autoimmune diabetes in adults: the Fremantle Diabetes Study. Diabetes Med 25(10):1245-1250. https://doi.org/ 10.1111/j.1464-5491.2008.02562.x

8. Lu J, Ma X, Zhang L, Mo Y, Ying L, Lu W, Zhu W, Bao Y, Zhou J (2019) Glycemic variability assessed by continuous glucose monitoring and the risk of diabetic retinopathy in latent autoimmune diabetes of the adult and type 2 diabetes. J Diabetes Investig 10(3):753-759. https://doi.org/10.1111/jdi.12957

9. Park H, Kim YG, Lee JW, Park JS (2014) Newly diagnosed diabetes mellitus patients presenting with proliferative diabetic retinopathy as an initial sign. Int J Ophthalmol 7(1):173-178. https://doi.org/10.3980/j.issn.2222-3959.2014.01.32

10. Expert Committee on the Diagnosis and Classification of Diabetes Mellitus (2003) Report of the expert committee on the diagnosis and classification of diabetes mellitus. Diabetes Care 26(Suppl 1):S5-20. https://doi.org/10.2337/diacare.26.2007.s5

11. Wilkinson CP, Ferris FL, Klein RE, Lee PP, Agardh CD, Davis M, Dills D, Kampik A, Pararajasegaram R, Verdaguer JT, Global Diabetic Retinopathy Project Group (2003) Proposed international clinical diabetic retinopathy and diabetic macular edema disease severity scales. Ophthalmology 110(9):1677-1682. https://doi. org/10.1016/S0161-6420(03)00475-5

12. Song P, Yu J, Chan KY, Theodoratou E, Rudan I (2018) Prevalence, risk factors and burden of diabetic retinopathy in China: a systematic review and meta-analysis. J Glob Health 8(1):010803. https://doi.org/10.7189/jogh.08.010803

13. Roh MO, Jung CH, Kim BY, Mok JO, Kim CH (2013) The prevalence and characteristics of latent autoimmune diabetes in adults(LADA) and its relation with chronic complications in a clinical department of a university hospital in Korea. Acta Diabetol 50(2):129-134. https://doi.org/10.1007/s00592-010-0228-y

14. Lu J, Hou X, Zhang L, Hu C, Zhou J, Pang C, Pan X, Bao Y, Jia W (2015) Associations between clinical characteristics and chronic complications in latent autoimmune diabetes in adults and type 2 diabetes. Diabetes Metab Res Rev 31(4):411-420. https://doi.org/ $10.1002 /$ dmrr.2626

15. Balme M, McAllister I, Davis WA, Davis TM (2002) Retinopathy in latent autoimmune diabetes of adults: the Fremantle Diabetes Study. Diabet Med 19(7):602-605. https://doi.org/10.1046/j.14645491.2002.00739.x

16. Cui J, Ren JP, Chen DN, Xin Z, Yuan MX, Xu J, You QS, Yang JK (2017) Prevalence and associated factors of diabetic retinopathy in Beijing, China: a cross-sectional study. BMJ Open 7(8):e015473. https://doi.org/10.1136/bmjopen-2016-015473

17. American Diabetes Association (2019) 11 Microvascular complications and foot care: standards of medical care in diabetes-2019. Diabetes Care 42(Suppl 1):S124-S138. https://doi.org/10.2337/ dc19-S011

18. Nusinovici S, Sabanayagam C, Teo BW, Tan G, Wong TY (2019) Vision impairment in CKD patients: epidemiology, mechanisms, differential diagnoses, and prevention. Am J Kidney Dis 73(6):846-857. https://doi.org/10.1053/j.ajkd.2018.12.047

19. Sasso FC, Pafundi PC, Gelso A, Bono V, Costagliola C, Marfella R, Sardu C, Rinaldi L, Galiero R, Acierno C, Caturano A, de Sio C, De Nicola L, Salvatore T, Nevola R, Adinolfi LE, Minutolo R, NO BLIND Study Group (2019) Relationship between albuminuric CKD and diabetic retinopathy in a real-world setting of type 2 diabetes: findings from No blind study. Nutr Metab Cardiovasc Dis 29(9):923-930. https://doi.org/10.1016/j.numecd.2019.05.065

20. Edwards MS, Wilson DB, Craven TE, Stafford J, Fried LF, Wong TY, Klein R, Burke GL, Hansen KJ (2005) Associations between retinal microvascular abnormalities and declining renal function in the elderly population: the Cardiovascular Health Study. Am J Kidney Dis 46(2):214-224. https://doi.org/10.1053/j.ajkd.2005. 05.005

21. Pedro RA, Ramon SA, Marc BB, Juan FB, Isabel MM (2010) Prevalence and relationship between diabetic retinopathy and nephropathy, and its risk factors in the North-East of Spain, a population-based study. Ophthalmic Epidemiol 17(4):251-265. https://doi.org/10.3109/09286586.2010.498661

22. Wong TY, Coresh J, Klein R, Muntner P, Couper DJ, Sharrett AR, Klein BE, Heiss G, Hubbard LD, Duncan BB (2004) Retinal microvascular abnormalities and renal dysfunction: the atherosclerosis risk in communities study. J Am Soc Nephrol 15(9):24692476. https://doi.org/10.1097/01.ASN.0000136133.28194.E4 
23. Lee WJ, Sobrin L, Kang MH, Seong M, Kim YJ, Yi JH, Miller JW, Cho HY (2014) Ischemic diabetic retinopathy as a possible prognostic factor for chronic kidney disease progression. Eye (Lond) 28(9):1119-1125. https://doi.org/10.1038/eye.2014.130

24. Kramer CK, Retnakaran R (2013) Concordance of retinopathy and nephropathy over time in Type 1 diabetes: an analysis of data from the Diabetes Control and Complications Trial. Diabet Med 30(11):1333-1341. https://doi.org/10.1111/dme.12296

25. Zhou Z, Xiang Y, Ji L, Jia W, Ning G, Huang G, Yang L, Lin J, Liu Z, Hagopian WA, Leslie RD, LADA China Study Group (2013) Frequency, immunogenetics, and clinical characteristics of latent autoimmune diabetes in China (LADA China study): a nationwide, multicenter, clinic-based cross-sectional study. Diabetes 62(2):543-550. https://doi.org/10.2337/db12-0207

26. Hawa MI, Thivolet C, Mauricio D, Alemanno I, Cipponeri E, Collier D, Hunter S, Buzzetti R, de Leiva A, Pozzilli P, Leslie RD,
Action LADA Group (2009) Metabolic syndrome and autoimmune diabetes: action LADA 3. Diabetes Care 32(1):160-164c. https://doi.org/10.2337/dc08-1419

27. Blaslov K, Bulum T, Knežević-Ćuća J, Duvnjak L (2015) Relationship between autoantibodies combination, metabolic syndrome components and diabetic complications in autoimmune diabetes in adults. Endocrine 48(2):551-556. https://doi.org/10. 1007/s12020-014-0331-x

Publisher's Note Springer Nature remains neutral with regard to jurisdictional claims in published maps and institutional affiliations. 